\title{
Filosofía y Ciencia en el Positivismo Lógico: una mirada retrospectiva*
}

\section{Philosophy and Science in Logical Positivism: $A$ retrospective}

\author{
VALERIANO IRANZO \\ UNIVERSITAT DE VALÈNCIA
}

Recibido: 21/01/2020 Aceptado:19/02/2020

\section{RESUMEN}

El Positivismo Lógico a menudo ha sido presentado como una corriente filosófica monolítica obviando las profundas disensiones que hubo en su seno. El presente artículo pretende corregir esta visión. Los dos primeros apartados describen la constitución del movimiento y los elementos supuestamente nucleares de la concepción filosófica positivista. El tercer apartado discute las discrepancias sobre el papel de la filosofía entre los autores más representativos del movimiento (Schlick, Carnap y Neurath). Para concluir se sugiere que la vigencia del legado positivista reside no tanto en alguna tesis filosófica en particular, cuanto en su compromiso metafilosófico con valores como la precisión y la claridad.

PALABRAS CLAVE

\section{POSITIVISMO LÓGICO, SCHLICK, CARNAP, NEURATH, CONCEPCIONES METAFILOSÓFICAS}

* Este trabajo ha recibido subvención del Ministerio de Economía y Competitividad (Gobierno de España) a través del proyecto FFI2016-76799-P. Agradezco los comentarios de los evaluadores de la revista Contrastes, así como los de los participantes en el congreso "Herencia y actualidad del Positivismo Lógico", celebrado en Valencia el 10 de marzo de 2017, donde fue presentada una versión previa de este trabajo. 


\section{ABSTRACT}

Logical positivism has been usually presented as a monolythic philosophical trend even though fundamental disagreements among its members were a matter of fact. This paper is focused precisely on those internal discrepancies. The historical setting and the allegedly fundamental philosophical claims are dealt with in the first two sections. The third section is focused on the notorious discrepancies among Schlick, Carnap and Neurath on the role assigned to philosophy. Finally, I suggest that the legacy of Logical Positivism lies not so much in any particular philosophical thesis, but rather in its metaphilosophical commitment to values such as precision and clarity.

\section{KEYWORDS}

LOGICAL POSITIVISM, SCHLICK, CARNAP, NEURATH, METAPHILOSOPHICAL VIEWS

\section{Surgimiento y Difusión Del Positivismo LóGico: CONSIDERACIONES HISTÓRICAS.}

En LA CRONOLOGía DEL PositiviSMo LóGiCo cabe distinguir dos periodos claramente diferenciados. El primero, el más fecundo, transcurre desde la década de los años 20 hasta la muerte de su fundador, Moritz Schlick, en 1936; el segundo se prolonga desde ese momento hasta finales de los años 60, y abarca el lapso de tiempo en que los positivistas lógicos ejercen su magisterio, mayoritariamente en universidades norteamericanas.

En su primera fase el movimiento se constituye y consolida en dos núcleos. El más importante fue el «Círculo de Viena» (Wiener Kreis), agrupado en torno a la figura de Moritz Schlick. Schlick había hecho su doctorado en física teórica con Max Planck en Berlín. Pronto sus intereses se encaminaron hacia la filosofía. En 1922 obtuvo la cátedra de Ciencias Inductivas de la Universidad de Viena, previamente ocupada por Ernst Mach, y consiguió reunir un conjunto de jóvenes con intereses y formaciones muy diversos: filósofos, lógicos, matemáticos, físicos y científicos sociales. ${ }^{1} \mathrm{El}$ comienzo oficial del Círculo de Viena fue en 1929, año en el que, haciendo uso de una forma expresiva popularizada por las corrientes artísticas renovadoras de la época, el manifiesto, se publica «La concepción científica del mundo. El Círculo de Viena.», una breve declaración de principios filosófica, firmada por Rudolf Carnap, Hans Hahn y Otto Neurath, y dedicada a Schlick (v. Uebel, 2008).

1 Stadler (2001a) es un estudio histórico pormenorizado sobre el Círculo de Viena, tanto biográfico como en relación al contexto cultural y político. 
El segundo de los núcleos fundacionales del Positivismo Lógico fue el «Círculo de Berlín» (Die Gesellschaft für Philosophie Empirische), en torno a Hans Reichenbach, Kurt Grelling y Walter Dubislav, e integrado por filósofos y científicos como Carl Gustav Hempel, David Hilbert y Richard von Mises. ${ }^{2}$

En 1930 Carnap y Reichenbach se hicieron cargo de una revista titulada Annales der Philosophie cambiando su nombre por Erkenntnis. Bajo el auspicio de la Gesellschaft für Empirische Philosophie, asociada al Círculo de Berlín, y del Verein Ernst Mach de Viena, la revista funcionó entre 1930 y 1938, convirtiéndose en el órgano principal de difusión para los positivistas hasta el inicio de la segunda guerra mundial. ${ }^{3}$ Aparte de esto, durante esa fase los miembros del Círculo de Viena forjaron conexiones a nivel internacional con la escuela de lógicos polacos (A. Tarski, J. Lukasiewicz, ...), B. Russell, L. Wittgenstein o F.P. Ramsey, radicados en Inglaterra, filósofos norteamericanos como Ch. Morris, E. Nagel o W.v. Quine, entre otros..., y figuras de otros países europeos. ${ }^{4}$

En cuanto a la exportación del pensamiento positivista fuera del ámbito de estudiosos más o menos próximos, conviene mencionar un primer intento exitoso: el artículo escrito por Herbert Feigl y Albert Blumberg titulado «Logical Positivism - A New Movement in European Philosophy», publicado en el Journal of Philosophy en 1931, que se convirtió en la carta de presentación del movimiento ante la comunidad filosófica en lengua inglesa. No obstante, en este apartado sobresale, con mucho, el libro Language, Truth and Logic, de Alfred Julius Ayer, publicado en 1936, que pretendía ser un resumen de las tesis centrales defendidas por el Círculo de Viena en las diversas áreas de la filosofía. A corto plazo tuvo un efecto impagable: propagar las ideas de los positivistas lógicos en el mundo filosófico de habla inglesa, mediante un texto fácilmente accesible. A largo plazo, en cambio, lo que ni fue, ni aspiraba a ser, un estudio profundo y exhaustivo de la filosofía positivista, tuvo un efecto negativo, al ser tomado por muchos de sus lectores como la interpretación oficial del movimiento. Y es que, en realidad, en su libro Ayer tomaba partido por una línea de trabajo en particular, a saber, la preconizada por Schlick y su discípulo más próximo, Friedrich Waissmann, bien diferente de las propuestas del «ala izquierda» del Círculo de Viena - expresión acuñada por Ernest Nagel,

2 Para una visión panorámica de este grupo, v. Milkov y Peckhaus (2013).

3 Su último número salió en 1939, ya con un nuevo título: The Journal of Unified Science (Erkenntnis) (1939-1940). Refundada en 1975, Erkenntnis sigue funcionando en la actualidad.

4 Sobre las conexiones con la Escuela Polaca, v. Wolénski y Köhler (1999). Sobre la influencia del pragmatismo norteamericano en el Círculo de Viena -décadas antes Mach ya mantuvo contacto regular con William James y había impartido conferencias en Estados Unidos a instancias de este último-, v. los artículos de Ferrari y Uebel incluidos en Pihlströmm et. al. (2017). 
a su vuelta a EE.UU. en 1936 tras una estancia en Viena (Nagel, 1936)-, en la que se incluían Neurath, Carnap, Hahn y Philip Frank.

La difusión internacional de las ideas de los positivistas lógicos corrió pareja con su marginalización creciente en la propia Austria y en Alemania. La Verein Ernst Mach, una sociedad paralela al Círculo de Viena, instituida en 1929, con objeto de popularizar la «filosofía científica» y el manifiesto fundacional como parte de un programa educativo para adultos, fue clausurada por razones políticas en 1934, con la llegada del nazismo al poder. Tras la trágica muerte de Schlick, asesinado por un estudiante dos años después, y ante la presión creciente en el ámbito académico, y fuera de él, sobre la población judía, comienza una segunda fase en el Positivismo Lógico caracterizada por el éxodo. Como ocurrió con muchos otros intelectuales, científicos o artistas centroeuropeos, las figuras principales del movimiento se establecerán sobre todo en Estados Unidos. Es el caso de Carnap, Reichenbach, Hempel, Frank o Feigl. Waissman se instaló en Inglaterra, recalando en Oxford, después de pasar dos años en Cambridge.

En Estados Unidos la influencia del positivismo lógico fue enorme. Filósofos norteamericanos de primera fila, como W. v. Quine o Wesley Salmon, entre otros, tuvieron como maestros a figuras como Carnap o Reichenbach, respectivamente. Aunque según algunos en este proceso de integración, en el que el Positivismo Lógico entró en contacto directo con la tradición pragmatista norteamericana, el movimiento perdió gran parte de su carácter rompedor, pasando a representar el statu quo académico (v. el prefacio en Friedman 1999), el maridaje de ideas fue muy fructífero en términos globales. Por un lado, obligó a los propios positivistas a retocar sus posiciones; por otro, alumbró nuevas perspectivas que eclosionaron a finales de los años cincuenta y comienzos de los sesenta del siglo pasado. ${ }^{5}$

\section{UN MOVIMIENTO PLURAL.}

En 1991 se fundó en Viena el Institut Wiener Kreis, con el objetivo prioritario de rescatar y revisar el legado del Círculo de Viena. Bajo su patrocinio se han publicado en las últimas tres décadas muchos textos previamente inaccesibles, ediciones de obras completas, etc. La bibliografía secundaria sobre el Positivismo Lógico también se ha incrementado notablemente a partir de esa fecha. Todo este material confirma la imagen de un movimiento filosófico plural que admitió en su seno posiciones diversas, incluso abiertamente encontradas. Sin embargo, en demasiadas ocasiones la imagen que se presenta del movimiento es una simplificación inaceptable,

5 Sobre la inserción de los positivistas lógicos en el medio universitario y cultural de la filosofía norteamericana, v. Holton (1993), Butts (1999) y Hardcastle y Richardson (2003). 
en contraste con la pluralidad de perspectivas que lo integraron. ${ }^{6}$ Así, el Positivismo Lógico se ha identificado a menudo con una suerte de credo unitario cuyos elementos son:

\section{verificacionismo semántico + fundamentismo epistemológico + reduccionismo fisicalista}

El texto de Ayer ya mencionado (Lenguaje, verdady lógica) probablemente resultó crucial a la hora de difundir esta interpretación, a pesar de que es más que dudoso que algún autor positivista defendiera las tres tesis a la vez. Y es que la interpretación más extendida del Positivismo Lógico lo emparenta estrechamente con la tradición empirista británica, y en particular con el empirismo de talante fenomenista, que se remonta al menos hasta Hume y Berkeley, y que a comienzos del siglo XX tiene en Bertrand Russell a uno de sus principales representantes (v. por ejemplo Passmore 1957). Sin negar que hubo puntos de encuentro destacables, más en unas figuras que otras, entre el Positivismo Lógico y el empirismo británico, el asunto es que no fue ese el marco cultural y filosófico en el que se educaron los positivistas. Sus reflexiones arrancan desde la tradición germana; y con independencia de los derroteros posteriores, es en relación a este contexto cultural como cabe entender tanto la eclosión del Positivismo Lógico como su planteamiento teórico.

No hay que pensar, por otro lado, que esa imagen simplificadora, a la cual se añade a veces otro rasgo: la asepsia respecto al compromiso social y el conservadurismo político ${ }^{7}$ haya sido vehiculada exclusivamente por posiciones filosóficas en las antípodas del Positivismo Lógico, como podrían ser la Hermeneútica fenomenológica, la Teoría Crítica o las corrientes sociologistas más recientes en filosofía de la ciencia,... Un ejemplo criticable en este sentido es Karl Popper, quien asistió a las reuniones del Círculo de Viena y mantuvo afinidades importantes con este, aunque, como es bien sabido, jamás quiso ser considerado como uno de sus miembros. Sus críticas al inductivismo del Círculo de Viena, y por extensión a los positivistas lógicos, no siempre están basadas en opiniones que ellos habían defendido.

6 Esta percepción, junto con la idea de que la visión simplificadora ha acabado convirtiendo al Positivismo Lógico en un «hombre de paja», se encuentra, por ejemplo, en la introducción de Friedman (1999).

7 También aquí se emborrona demasiado la situación real. Los miembros del Círculo participaron activamente en el estimulante ambiente artístico e intelectual de la Viena de la época, promoviendo iniciativas culturales concretas, como la Verein Ernst Mach y, en algunos casos, también a través de la militancia política. Sobre estas cuestiones v. Stadler (2001b), Uebel (2012) y Cartwright et al. (1996). 
Desde luego, la obra de Popper es mucho más que un alegato anti-inductivista, pero con su actitud contribuyó a forjar esa interpretación simplificadora e interesada (v. Koterski, 2012).

El hecho es que, a poco que se estudie la evolución de las figuras principales del movimiento, se comprueba, primero, que el contexto del que arranca la discusión de los positivistas lógicos no es el propio de la tradición empirista británica, lo que no es sorprendente; y segundo, que ejercieron la autocrítica con sus compromisos filosóficos, incluyendo los de la terna indicada. Las dos subsecciones que siguen pretenden socavar, pues, aquella imagen monolítica y sesgada del movimiento.

\section{II.1 LaS Raíces fiLosóficas del Positivismo Lógico.}

El proyecto de elaborar una «filosofía científica» a principios del siglo XX no era algo novedoso en la tradición filosófica germana. Uno de sus adalides fue Hermann von Hemlholtz, destacado sobre todo por sus aportaciones en fisiología y psicología, pero cuyos intereses se extendieron al campo de la filosofía. A mediados del siglo XIX, y ante los excesos especulativos de la Naturphilosophie de Hegel y Schelling, Hemlholtz proclamó la necesidad de una wissenschaftliche Philosophie, una filosofía que trabaje en cooperación con la ciencia. Esto se presenta, además, como una vuelta al espíritu de la obra de Kant, pues se consideraba que esta ejemplificaba ese espíritu integrador. A fin de cuentas, en la Crítica de la Razón Pura el referente modélico del conocimiento es la física newtoniana. Según Helmholtz, urgía en ese momento repensar la visión kantiana del problema sujeto/objeto de acuerdo con los descubrimientos recientes de la ciencia, y en especial con los realizados por la psicofisiología, campo en el que las aportaciones del propio Helmholtz fueron relevantes. ${ }^{8}$

Helmholtz ejemplifica este neokantismo «científico», que insiste en desvincular la filosofía de las discusiones metafísicas, porque las considera infundadas y decepcionantes, para convertirla en una epistemología o teoría de la ciencia. Este planteamiento tuvo su momento álgido en las décadas de 1870 y 1880, a través de figuras con notable impacto en los círculos intelectuales y científicos centroeuropeos (Richard Avenarius, por ejemplo). Pero con el cambio de siglo el neo-kantismo sufrió una reorientación de manos de las escuelas neokantianas de Marburgo (Hermann Cohen, Paul Natorp y Ernst Cassirer) y del Suroeste (Heinrich Rickert y Wilhem Windelband). Aunque se mantenía el interés por las ciencias naturales, la reapropiación de Kant

8 En esta breve semblanza del planteamiento de Hemlholtz he seguido a Friedman (2012). 
consistía ahora más bien en discutir las presuposiciones metafísicas de la ciencia y en insistir, además, en las peculiaridades de las ciencias humanas frente a las naturales.

La formación de muchos de los positivistas lógicos fue mixta, en ciencia y en filosofía. En todo caso, la influencia filosófica directa que recibieron fue la del neokantismo no-científico, predominante en el medio académico germano, bien a través de los profesores de sus años de formación, bien a través de la discusión subsiguiente en sus años de juventud. ${ }^{9}$ Desde luego, los positivistas pronto se distanciarán de esa posición neokantiana, pero el esquema teórico de partida es deudor de ella. Ninguna de las figuras principales del movimiento parte de la problemática propia del empirismo fenomenista. En cambio, la distinción entre forma y contenido del conocimiento, que se resuelve desde una perspectiva kantiana en los juicios sintéticos a priori, ocupa un lugar central en las reflexiones de Schlick, Carnap o Reichenbach anteriores al manifiesto «La concepción científica del mundo». Y debe tenerse en cuenta que es en ese periodo cuando se escriben obras fundamentales como Allgemeine Erkenntnislehre de Schlick en 1918, Relativitatstheorie und Erkenntnis A Priori de Reichenbach en 1920, o Der Logische Aufbau der Welt de Carnap en $1928 .{ }^{10}$

El edificio kantiano no podía seguir incólume frente a los descubrimientos científicos realizados a finales del siglo XIX. Concretamente, la axiomatización de la geometría por parte de Hilbert -considerada como ejemplo de sistematización y objetivación del conocimiento científico- y la teoría de la Relatividad de Einstein, plantearon dudas sobre el supuesto carácter necesario del espacio plano, euclídeo, y de las leyes principales de la mecánica newtoniana. La intuición kantiana -una intuición pura que fija el

9 Para una discusión detallada de la relación entre Schlick y Carnap y el neokantismo, pueden verse los caps. 1 y 6 de Friedmann (1999). En cuanto a las influencias filosóficas recibidas por Carnap, v. Carus (2008).

10 Aunque el Aufbau ha sido a menudo considerado un sofisticado ejercicio de empirismo fenomenista, y las lecturas de Quine y Goodman en los años cuarenta y cincuenta del siglo pasado así lo han presentado, en su contexto no se planteó como tal. Carnap intentó construir la dimensión heteropsicológica a partir de una base psicológica, subjetiva, la relación del «recuerdo de la semejanza» (̈̈hnlikcheitserinnerung) entre vivencias, que son experiencias globales del sujeto. Pero la razón que Carnap da en el propio Aufbau para preferir una base autopsicológica es instrumental. Así, la metodología construccional podía haberse desarrollado al revés, con lo cual lo realmente importante es dicha metodología lógica, o cuasi-lógica, comprometida con la neutralidad ontológica y con la idea de que el significado de un concepto no depende tanto de su contacto con lo empíricamente dado, sino de las relaciones formales y estructurales con el resto de conceptos que integran el sistema. A favor de esta interpretación, v. Friedman (1999) cap. 5, y los capítulos 4 al 7 de Richardson (1998). V. también Moulines (1996) para matizar los compromisos fenomenistas y empiristas del Aufbau. 
marco espacial y temporal en que acontecen los fenómenos- será descartada por nuestros autores; el proyecto de una «lógica trascendental», que aspire a establecer condiciones necesarias de posibilidad de la experiencia objetiva, es rechazado de plano. En la línea de filosofar teniendo en consideración la ciencia del momento, recuperando el lema de la filosofía «científica» de Helmholtz, se apostará por la relativización del aparato apriorístico kantiano y el deslizamiento hacia posiciones convencionalistas (la influencia del matemático y filósofo francés Henri Poincaré fue aquí notable). Esta es la senda que Schlick, Carnap y Reichenbach transitarán, cada uno a su modo, durante el periodo que nos ocupa.

En suma, el empirismo fenomenista ni jugó un papel preponderante en el contexto formativo de los positivistas lógicos, ni se planteó como posicionamiento filosófico en el surgimiento del movimiento. Su importancia, en todo caso, crece después, a partir de 1929 al socaire de la interpretación particular que algunos miembros del Círculo de Viena, en especial Schlick, hacen del Tractatus Logico-Philosophicus de Wittgenstein.

\section{II.2 ENCUENTROS Y DISENSIONES.}

Elhecho es que en el propio Círculo de Vienahubodesacuerdos importantes. Antes nos hemos referido al «ala izquierda» del Círculo (Neurath, Carnap, Hahn y Frank). Sus integrantes discrepaban de M. Schlick y F. Waismann; en concreto, sobre la valoración del Tractatus Logico-Philosophicus de Wittgenstein. Schlick estuvo fuertemente influenciado por esta obra y, de hecho, Wittgenstein fue invitado a varias sesiones del seminario que celebraba el Círculo de Viena. Schlick pretendía complementar el Tractatus con una epistemología empirista: suponiendo que el armazón metafísico del mundo sea una reproducción casi especular de la estructura lógica del lenguaje, tal como afirma el Tractatus, ¿cómo podemos obtener conocimiento sobre aquél? El «principio de verificación», entendido como un criterio de significado, jugaba aquí un rol crucial. En cambio, el «ala izquierda» era partidaria de desligar el criterio de significado de la verificación. Además, el «ala izquierda» no compartía el escepticismo wittgensteniano sobre la noción de metalenguaje (condensado en la distinción tractariana entre 'decir' y 'mostrar'), y estaba contra una posición «fundamentista» del conocimiento, por mucho que esto se pretendiera defender apelando a una base de carácter empírico pretendidamente incorregible. De hecho, a mediados de los años 30 Carnap y Neurath renegaron explícitamente del fundamentismo epistemológico y apostaron por teorías coherentistas de la verdad.

Y si dentro del mismo Círculo de Viena ya hubo desacuerdos, cómo no iban a producirse estos en relación a las posiciones defendidas por otros positivistas pertenecientes a otros grupos. Un botón de muestra es la 
publicación en 1936 por parte de Reichenbach, líder del Círculo de Berlín, de una suerte de contra-manifiesto, «Logistic Empiricism in Germany and the Present State of Its Problems», en respuesta al escrito fundacional de 1929.

Pero entonces, ¿es que no podría reconocerse alguna tesis genérica compartida por todos los miembros del movimiento? Si hubo tal cosa, a la hora de darle concreción surgieron diferencias tan notables que el contenido realmente compartido apenas quedó en un eslogan. Un ejemplo podría ser la unidad de la ciencia (Einheitwissenchaft), con la cual los positivistas lógicos se posicionaban en una enconada disputa académica, que se arrastraba desde mediados del siglo XIX, frente a la supuesta especificidad de las «ciencias humanas o sociales» (Geistwissenchaften), punto este sobre el que la escuela neokantiana del Suoreste era particularmente combativa. Pero más allá de eso hubo divergencias importantes sobre cómo entender la unidad de la ciencia. Mientras algunos positivistas optaron por desarrollar una estrategia «construccionista» (un ejemplo sería el Aufbau de Carnap), mostrando cómo todos los conceptos científicos están interconectados, otros interpretaron la tesis más bien como parte de un programa social y político, es el caso de Neurath, de modo que para este último el objetivo de la unidad de la ciencia tenía más que ver con el activismo que con la reflexión puramente teórica, como se verá en el siguiente apartado.

También compartieron los positivistas lógicos una acentuada desconfianza para con las disquisiciones metafísicas, siendo esta, seguramente, la seña de identidad del movimiento de cara a la posteridad, quizá por ser la más llamativa y polémica. Así, en el primer número de Erkenntnis, el órgano de difusión de los positivistas, uno de sus editores, Reichenbach, destacaba como objetivo programático de la revista acabar con la filosofía entendida como una sucesión de «sistemas filosóficos» para convertirla en un conocimiento objetivo.

Ciertamente, en torno a 1920 esto ya no podía considerarse una novedad. Hume, Kant, Comte, los pragmatistas norteamericanos y los neo-kantianos «científicos» ya habían manifestado su abierto desencanto ante el supuesto conocimiento que la metafísica había aportado a lo largo de la historia. No obstante, aunque una motivación subyacente a la reflexión de los positivistas era la desesperanza ante la imposibilidad de constatar algún tipo de avance en la solución de los problemas metafísicos, la explicación de cuál es el problema de fondo que impide progresar no fue unitaria. Una de las herramientas empleadas para criticar las pretensiones de la metafísica fue, ciertamente, el principio de verificación, entendido como un criterio de significado. La exigencia de la verificación empírica convertía al discurso metafísico en un discurso carente de significado, racionalmente ininteligible, por más que pudiera tener quizá algún significado «no cognitivo», como el arte tal vez. La metafísica no era, pues, un conjunto de afirmaciones falsas; era un conjunto de afirmaciones que 
no podían ser ni verdaderas ni falsas. De ella no quedaría entonces más que un vano intento de obtener un conocimiento «supra-empírico», conocimiento que se revela imposible, de la realidad.

Schlick y Waismann siguieron por este camino, en un intento de elaborar una epistemología para el Tractatus de Wittgenstein. Pero el diagnóstico y la cura antimetafísica preconizada por otros positivistas iba en otra dirección. Para Carnap, por ejemplo, la corrección de un lenguaje no dependerá de su coincidencia con la estructura íntima del mundo. ${ }^{11}$ La creatividad en la invención de lenguajes formales no debe verse coartada porque la justificación de uno u otro es puramente pragmática. ${ }^{12}$ En The Logical Syntax of Language, publicado en 1934, Carnap intentó disolver las disputas metafísicas a través de la distinción entre «modo formal» $\mathrm{y}$ «modo material» de hablar. El primero se articula con oraciones sobre el lenguaje; el segundo cuando aparentemente se habla sobre eventos, procesos o cosas. Esta distinción permitirá a Carnap reconvertir las discusiones metafísicas en discusiones sobre modos de hablar o preferencias lingüísticas, relativizando así la ontología al lenguaje.

Pero, más allá de la convicción compartida entre los positivistas lógicos de que la metafísica es un lastre, cabe reconocer que, primero, la crítica de la metafísica se hace desde la filosofía; y segundo, que aquella convicción deja un amplio margen para discutir cuál era el papel que correspondía a la filosofía. Y es que la tesis de que la eliminación de la metafísica comportaba necesariamente la desaparición de la filosofía in toto, como si tras la denuncia de la metafísica nada filosófico quedara por decir, fue defendida, si es que lo fue, por una exigua minoría (quien más se acercó a ello seguramente fue Neurath). Sobre el asunto capital de definir los objetivos y metodología de la reflexión filosófica se advierten diferencias sustanciales entre los positivistas, como veremos en el siguiente apartado, en el que se comparan las ideas de tres figuras tan relevantes como Schlick, Carnap y Neurath.

\section{Filosofía y Ciencia en el Positivismo LóGico.}

Al poco de la publicación del Manifiesto de 1929, Schlick describió qué competencias correspondían a la filosofía en la era «post-metafísica». Tras referirse al «estéril conflicto entre los sistemas filosóficos», sostuvo que ni hay «verdades filosóficas», ni la filosofía es un sistema de proposiciones,

11 Sobre los puntos de acuerdo y desacuerdo por parte de Carnap respecto al Tractatus, v. Carnap (1934a), 282-284 y Friedman (1999), cap. 8.

12 El Principio de Tolerancia de Carnap reza así: «In logic, there are no morals. Everyone is at liberty to build up his own logic, i.e. his own form of language as he wishes. All that is required of him is that, if he wishes to discuss it, he must state his methods clearly, and give syntactical rules instead of philosophical arguments.» (Carnap, 1934a, 52). 
ni tampoco una ciencia. Sin embargo, esto no implica la eliminación de la filosofía:

[...]; philosophy, in fact, is that activity whereby the meaning of statements is established or discovered. Philosophy elucidates propositions, science verifies them. In the latter we are concerned with the truth of statements, but in the former with what they actually mean. The content, the heart and soul of science, is naturally located in what its propositions ultimately signify; the philosophical activity of giving significance is thus the alpha and omega of all scientific knowledge. ... philosophy furnishes both the foundation and the summit to the edifice of science; the only error has been to suppose the foundation to consist of 'philosophical propositions' (the propositions of the theory of knowledge) and the building also to be crowned by a dome of philosophical propositions (called metaphysics).(Schlick, 1930/1979b, 157) ${ }^{13}$

Schlick atribuye a la filosofía una función básicamente elucidatoria. Y no solo es una actividad legítima; es imprescindible. Según él, la ciencia no se sostiene por sí misma; necesita el concurso de la filosofía que es la encargada de justificar/mostrar el significado/sentido de aquella. Aunque es cierto que Schlick remarca la dimensión activa de la filosofía, quizá por influencia de Wittgenstein, es obvio que cualquier actividad requiere la aplicación de ciertos recursos. Por eso la filosofía no es solo esclarecimiento conceptual, eso sería más bien el resultado que se persigue. La filosofía es también la reflexión sobre, y la elaboración de, esas herramientas teóricas que permiten el ejercicio de la actividad elucidatoria.

En esta línea Schlick dedicó sus esfuerzos a refinar el criterio positivista del significado, el Principio de verificación, que él rebautizó como «Principio de Verificabilidad», para dejar claro que lo que importaba no era tanto la verificación efectiva de una proposición como su posibilidad de ser verificada (Schlick, 1936/1979b). También se ocupó de la caracterización de las proposiciones empíricas básicas sobre las que se asienta el discurso cognitivamente significativo, las famosas ProtoköllSatze, que él identificó con una suerte de vivencias del sujeto, las 'constataciones' [Konstantierungen] (v. Schlick, 1934/1979b).

No obstante, a pesar de la novedad que la propuesta metafilosófica de Schlick en el contexto filosófico centroeuropeo de comienzos del siglo XX, convirtiendo a la filosofía en una actividad elucidatoria, la suya fue la más conservadora en relación a lo planteado en el Círculo de Viena, en el sentido de la más continuista con la tradición filosófica. Aun compartiendo el objetivo común de la «liberación» de la metafísica, y aunque el resultado de sus

13 La paginación de las citas de Schlick y Neurath en esta sección es la de los Philosophical Papers sobre cada uno de ambos autores incluidos en la bibliografía final. 
reflexiones le llevara a negar que el discurso científico necesita un basamento adicional proporcionado por la filosofía. Schlick siguió en los años treinta cultivando la Teoría del Conocimiento en un sentido tradicional, como había hecho hasta entonces, si bien virando hacia un fundamentismo epistemológico de corte empirista, un viejo conocido en la historia de la filosofía.

En este punto es digno de reseñar el contraste entre Schlick y Carnap. A comienzos de los años treinta el distanciamiento entre ambos es patente:

In order to discover the correct standpoint of the philosopher, which differs from that of the empirical investigator, we must not penetrate behind the objects of empirical science into presumably some kind of transcendent level; on the contrary we must take a step back and take science itself as the object. Philosophy is the theory of science. Philosophy deals with science only from the logical viewpoint. Philosophy is the logic of science, the logical analysis of the concepts, propositions, proofs, theories of science, both of those occurring in existing science as well as of those which are common to the possible methods of constructing concepts, proofs, hypotheses, theories. (Carnap, 1934b)

Aunque Carnap insiste en delimitar una parcela específica para la filosofía, rechaza la pertinencia de la teoría del conocimiento tradicional al identificar la filosofía con la lógica de la ciencia [Logikwissenchaft], pues no parece que el análisis lógico del lenguaje científico permita ya no pronunciarse, sino ni siquiera plantear cuestiones epistemológicas centrales como los límites del conocimiento humano, la relación sujeto/objeto,... Además, Carnap apuesta resueltamente por transformar la filosofía en un discurso metacientifico, opción con la que Schlick, que atribuye a la filosofía el análisis del significado de conceptos no necesariamente científicos, no simpatizaría especialmente.

Lo que el análisis lógico muestra es que la metafísica no aporta ningún conocimiento, ni empírico ni supraempírico, de la realidad porque, pese a las apariencias, en ella no hay afirmaciones de hecho, sino recomendaciones lingüísticas. En Carnap (1934a) parte V, se diferencia entre oraciones-objeto ('5 es un número primo'), oraciones-pseudo-objeto (' 5 es un número, no una cosa') y oraciones sintácticas ('5 no es una palabra-cosa, sino una palabranúmero'). El modo material de hablar está integrado por oraciones-pseudoobjeto; el modo formal por oraciones sintácticas. El modo material es en el que usualmente se expresan los filósofos. Según Carnap, la traducción del modo material al modo formal de hablar evita que se generen «pseudoproblemas», «i.e.: questions which, because of a misleading formulation, appear to refer to objects while actually they refer to sentences, terms, theories and the like» (ibid., 281). Fenomenistas, idealistas, realistas,.., disputan entre sí, pero desenmascarar las pretensiones de la metafísica, según Carnap, pasa por 
mostrar que tales disputas no son acerca de cómo es la realidad, sino acerca de cómo hablar de ella. La futilidad epistemológica de la metafísica se sigue directamente de aquí. ${ }^{14}$

A golpe de vista al menos, la posición de Carnap resulta bastante restrictiva. ¿Es que no queda entonces espacio para la epistemología (la teoría del conocimiento)? ¿Debe esta correr el mismo destino que la metafísica? La respuesta exige matizaciones.

Digamos primero que la propuesta de Carnap en su (1934a) mantiene cierta continuidad con la ocupación tradicionalmente reservada a la filosofía, a saber, la articulación de un discurso a priori independiente de la información empírica que posee cierto carácter normativo (en este caso, normatividad epistemológica). Esta idea se mantendrá en el resto de su obra. Carnap aplicará en los años 50 y 60 su idea de la filosofía como «lógica de la ciencia» en la elaboración de su «lógica inductiva»(Carnap, 1950/1962). Si la inferencia deductiva suponía una «implicación total» entre proposiciones, dado que la verdad de las premisas necesariamente exige la verdad de la conclusión, la inferencia inductiva debía verse como una «implicación parcial» en la que las premisas - la evidencia- hacían más o menos probable la conclusión la hipótesis que ha de ser apoyada empíricamente. Carnap desarrolló así la interpretación lógica, o «logicista», de la probabilidad (previamente defendida por John M. Keynes), en oposición a otras interpretaciones (frecuencialista, bayesiano-subjetivista,..).

Pero lo que más nos interesa destacar aquí es que Carnap veía este proyecto como una «reconstrucción racional», una suerte de epistemología «pura», netamente distinguible de una epistemología «psicológica». Al inicio de The Logical Concept of Probability, Carnap deja claro que la filosofía -la epistemología «pura»- tiene una tarea positiva y específica a la que se refirió con un término ad-hoc [explication]:

The task of explication consists in transforming a given more or less inexact concept into an exact one or, rather, in replacing the first by the second. We call the given concept (or the term used for it) the explicandum, and the exact concept proposed to take the place of the first (or the term proposed for it) the explicatum. The explicandum may belong to everyday language or to a previous stage in the development of scientific language. The explicatum must be given by

14 Vaya un ejemplo. El conflicto entre positivismo ('una cosa es un grupo de datos sensoriales') y realismo ('una cosa es un grupo de atomos'), dos tesis filosóficas incompatibles, se disuelve cuando traducimos cada una de ellas al modo formal de hablar (respectivamente: 'Toda oración en la que un término-cosa ocurre es equipolente a una clase de oraciones en la cual no hay ninguna que designe cosas, sino datos sensoriales' y 'Toda oración en la que un término-cosa ocurre es equipolente a una clase de oraciones en la que hay coordenadas espacio-temporales y functores descriptivos de la física'). La contradicción desaparece. (Carnap, 1934a, 301) 
explicit rules for its use, for example, by a definition which incorporates it into a well-constructed system of scientific either logico-mathematical or empirical concepts. (Carnap, 1962, 6)

Desde luego, explication no puede traducirse por 'explicación', sin más; de hecho, no debe confundirse con otro término parecido como 'explanation'. 'Explication' es el término elegido por Carnap para referirse al análisis conceptual, algo practicado propiamente por el filósofo, aunque no solo por este, pues también los científicos y los matemáticos ocasionalmente se ocupan de ello. Por lo demás, para articular el explicatum Carnap prefiere recurrir a un lenguaje formalizado, antes que al lenguaje natural. Sugiere también varios criterios para determinar cuándo el explicatum es adecuado en relación a un explicandum, aunque admite que no debe hablarse de aceptabilidad o corrección del explicatum en términos absolutos. Dada la vaguedad inevitable en los datos de los que se parte (es decir, los contextos de uso, los ejemplos paradigmáticos, etc., relativos al explanandum), serán más bien consideraciones pragmáticas las que permitan inclinar la balanza a favor de alguno de los análisis [explications] alternativos. (Carnap 1962, pp. 5-6)

Por último, y para abundar en la pluralidad de perspectivas dentro del Positivismo Lógico, comentaremos las ideas de Otto Neurath, científico social y marxista. Neurath fue sin duda un filósofo poco convencional. Activista y visionario, no desarrolló sus propuestas con la profundidad teórica con que lo hicieron otros compañeros del movimiento, pero no por eso fueron menos sugerentes (v. Cat et al. (1995) y Cartwright et al. (1996)). Neurath fue también uno de los miembros del Círculo de Viena más hostiles a la metafísica. Su actitud quedó manifiesta en las reuniones del Círculo a las que asistió Wittgenstein. ${ }^{15}$ Como Carnap, Neurath apostó claramente en contra de la teoría del conocimiento tradicional, lo que significaba posicionarse frente a Schlick:

[...] within a consistent physicalism there can be no 'theory of knowledge', at least not in the traditional form. It could only consist of defence actions against metaphysics, i.e.: unmasking meaningless terms. Some problems of the theory of knowledge will perhaps be transformable into empirical questions so that they can find a place within unified science. (Neurath, 1931b/1983, 67)

De la teoría del conocimiento nos interesan exclusivamente las cuestiones que puedan ser abordadas empíricamente, lo que aquí es sinónimo de 'científicamente'. La filosofía debe consagrarse exclusivamente a denunciar

15 La complicada relación entre Wittgenstein y el Círculo de Viena -tampoco congenió con Carnap-, ha dado para muchas páginas. Un relato escrito por uno de los participantes en las reuniones es Waismann (1973). La visión del propio Carnap se encuentra en Carnap (1992). 
la carencia de significado de aquellas cuestiones que no sean reducibles a la ciencia. Esta es una actividad meramente «defensiva» porque, según Neurath, no hay lugar ya para la construcción de un corpus de verdades filosóficas.

En esta línea, la Enciclopedia de la «Ciencia Unificada», proyecto editorial al que Neurath se consagró hasta su fallecimiento en 1946, pretendía ser una síntesis de los avances que la ciencia, natural y social, había conseguido hasta el momento. The International Encyclopedia of Unified Science, coeditada por él junto con Rudolf Carnap y Charles Morris (v. supra nota 3), era la contraparte positivista a los sistemas metafísicos, esto es, la imagen científica de la realidad, imperfecta y revisable, pero con aspiraciones omnicomprensivas, como las de aquellas cosmovisiones filosóficas:

Built up with the help of the logic of science, unified science replaces a comprehensive view as it was attempted by metaphysics, by a planned synthesis of everything that we have produced in the way of scientific statements. (1936a/1983, 132).

Neurath criticó la metafísica, igual que sus compañeros de viaje del Círculo de Viena, por su carácter confuso y ambiguo. A este propósito llegó incluso a sugerir la creación de una disciplina denominada «Terminología» [Terminology] (Neurath, 1941) para filtrar aquellos términos que no tienen cabida en el lenguaje fisicalista de la Ciencia Unificada. Su arma contra la metafísica será, pues, el fisicalismo, tesis que afirma que: «...terms similar to those employed in physics and in our everyday language are sufficient for constructing all sciences.» (Neurath 1937, 176).

En realidad, lo que determina el significado para Neurath poco tiene que ver con la verificación/verificabilidad al modo de Schlick. La traducibilidad al lenguaje fisicalista de la Ciencia Unificada es el criterio de significado. Entre los términos filosóficos que no son admisibles en dicho lenguaje y que, por tanto, quedan fuera de la Ciencia Unificada están: 'existencia', 'entidad', 'realidad', 'cosa', 'hecho', 'concepto'. 'mente'. «mundo mental', 'mundo físico', 'significado', 'progreso', 'bueno/malo', 'significado', 'progreso', 'lo bello’ y ‘lo bueno' (Neurath, 1944, cap. 1, secc. 7). Es por esta razón por la que Neurath considera que la metafísica y gran parte de la teoría del conocimiento carecen de significado. ${ }^{16}$

Aunque esto parece aproximarse al planteamiento de Carnap, Neurath insistió en lainsuficiencia de un enfoque formalista, en oposición al sintacticismo

16 Neurath dice que muchos términos filosóficos no tienen significado, incluyendo el propio término 'significado'. No hay contradicción aquí porque Neurath se refiere a la concepción filosófica tradicional mentalista que equipara los significados a conceptos. Lo que se está diciendo, pues, es que el término 'significado', en su acepción filosófica tradicional, no tiene significado, en su acepción fisicalista. 
defendido por Carnap. Neurath recalcó la necesidad de complementarlo atendiendo a la conducta de los investigadores (behavioristics of scholars), esto es, al proceso fáctico que desemboca en la aceptación/rechazo de las teorías científicas teniendo en cuenta que ello debe contar ineludiblemente con factores externos, no solamente físicos, biológicos o psicológicos, sino también con el contexto social y económico.

Frente a una posición que atendía exclusivamente a la dimensión lógicoepistemológica de la ciencia, Neurath trataba de colocar en el punto de mira los criterios de aceptabilidad, tanto de las teorías científicas como de los propios datos observacionales, puesto que sobre estos últimos siempre defendió una posición convencionalista. Ante un conflicto entre un enunciado protocolar (un enunciado que registra los datos observables) y un enunciado teórico, la anulación del enunciado protocolar es una opción metodológica totalmente legítima (Neurath, 1932a). La verdad se convierte, para él, en un asunto de coherencia intralingüística (v. Neurath, 1931a), mientras que la probabilidad se reserva exclusivamente para el cálculo matemático, con lo que no hay lugar para una teoría probabilística de la confirmación en la línea carnapiana (Neurath, 1941, 222). La plausibilidad/aceptabilidad de las teorías científicas no es un asunto puramente epistemológico, que dependa exclusivamente del apoyo evidencial que la observación les confiere, sino pragmático. El factor crucial aquí es la relación entre el conglomerado teoría-observación y un agente colectivo, la comunidad de investigadores, que establece los criterios para la toma de decisiones según cuáles sean los objetivos que se persiguen, y que está inevitablemente condicionada por constricciones externas a la propia ciencia. La normatividad inherente a este proceso sería un resultado de su dinámica interna, más bien que una imposición desde una perspectiva filosófica externa.

La Ciencia Unificada se construye contando con la lógica de la ciencia, como afirma Neurath en la cita anterior, pero no se reduce, ni mucho menos, a ella. Por otro lado, Neurath siempre remarcó el carácter provisional, revisable, de la Enciclopedia, lo que lleva a destacar la impronta naturalista de su planteamiento, anticipándose a un estilo filosófico que se pondría en boga décadas después de su muerte y que ejerce una influencia notable a día de hoy. Una de las notas definitorias del naturalismo epistemológico es que cuestiona la posibilidad de una perspectiva externa al conocimiento desde la cual justificarlo globalmente. Neurath recurrió al símil del barco, popularizado años después por Quine: el conocimiento es como un barco que ha de ser reparado en alta mar; no podemos desmontarlo y reconstruirlo por entero, como si estuviera en un dique seco, porque nos hundiríamos; hemos de hacerlo por piezas (Neurath, 1932a, 92-3). Con otras palabras, no 
es posible articular un discurso separado y distinto de la ciencia que aporte criterios de justificación o racionalidad extrínsecos a esta.

En consonancia con esto, la Ciencia Unificada no puede ser sino un discurso metacientífico, esto es, sobre la propia ciencia, que aporta conocimiento genuino acerca de esta. La Ciencia Unificada contiene dos dimensiones: una empírica, en la que se incluyen la historia de la ciencia y la sociología de la ciencia, entendidas ambas en clave fisicalista; y otra constituida por la pragmática de la ciencia, esto es, la conducta de los investigadores [behavioristics of scholars] (v. Neurath, 1932b y 1936b). Neurath, filósofo pero también científico social, insiste en que la ciencia debe expresarse en un lenguaje fisicalista, expurgado de aditamentos metafísicos, pero también subrayará el carácter grupal y cooperativo de la empresa científica.

Esta visión hace patente su oposición tanto al fundamentismo epistemológico de Schlick, en el que los enunciados basados en percepciones y vivencias del sujeto individual se convierten en el suelo rocoso que fundamenta el conocimiento científico, como a la epistemología a priori de Carnap, probabilista y formalista, ${ }^{17}$ centrada exclusivamente en las relaciones de apoyo confirmacional entre enunciados. Para Neurath, en cambio, teorizar sobre la ciencia es teorizar sobre la sociedad; la objetividad, la racionalidad, los valores que orientan la actividad científica, son de naturaleza social, no individual. Además, la ciencia debe convertirse en un factor liberador, en un instrumento para la reforma social, subrayando así la importancia de la dimensión activista o transformadora, una dimensión que para él fue motor y consecuencia a la vez de su reflexión teórica.

\section{El Legado del Positivismo LóGico.}

La comparación efectuada en la sección anterior muestra desacuerdos fundamentales entre las figuras señeras del Positivismo Lógico en un asunto nuclear como es la tarea y los objetivos de la reflexión filosófica. Esto es un síntoma de la riqueza y pluralidad de perspectivas desarrolladas en el seno del movimiento. Casi un siglo después seguramente no encontraremos ningún filósofo que se autoproclame "positivista lógico", sin más cualificaciones. Si

17 Neurath reconoció que su perspectiva, la «prágmática de la ciencia», que a día de hoy calificaríamos de naturalista sin ambages, podría ser complementada con la «lógica de la ciencia», en el sentido carnapiano visto antes, ocupada con cuestiones sintácticas y semánticas. Carnap, por su parte, también se manifestó a favor de la complementariedad. Dada la disparidad de los planteamientos, y dado que ninguno de los dos se implicó en una reflexión teórica integradora, cabe pensar que estamos ante declaraciones recíprocas de cortesía. Thomas Uebel, sin embargo, ha defendido que dicha complementariedad es posible: «Carnap's and Neurath's cooperation was not always harmonious and in their theoretical emphases they often differed, but in their goal they always agreed» (Uebel, 2018, 381; v. también Uebel, 2015). 
así fuera estaríamos ante una figura anacrónica. Y es que, si el inicio oficioso del Positivismo Lógico podríamos datarlo en 1929, fue justamente un positivista lógico quien fechó su defunción, cuarenta años después, según cuenta uno de los presentes en ese momento, el filósofo de la ciencia norteamericano Frederick Suppe:

The Received View on Theories was the epistemic heart of Logical Positivism. Twelve hundred persons were in the audience the night it died. It was March 26, 1969-opening night of the Illinois Symposium on the Structure of Scientific Theories. The Received View had been under sustained attack for a decade and a critical mass of main protagonists had been assembled to fight it out. Carl Hempel, a main developer of the Received View, was the opening speaker and was expected to present the Received View's latest revision. Instead he told us why he was abandoning both the Received View and reliance on syntactic axiomatizations. Suddenly we knew the war had been won, and the Symposium became an energized exploration of where to go now. (Suppe, 1998)

Después de esto, después de que uno de los máximos artífices de la concepción de la ciencia del Positivismo Lógico (la llamada «Concepción Heredada» [the Received View]), declarara su defunción, ¿puede decirse, pues, que alguna de las ideas o proyectos centrales del Positivismo Lógico sea hoy en día defendible?

Las historias de la filosofía suelen incluir el Positivismo Lógico como un «aggiornamiento» del empirismo clásico dentro de lo que se ha dado en llamar «el giro lingüístico» [the linguistic turn], un momento filosófico característico del siglo XX (Rorty, 1967). Un presupuesto metafilosófico básico del giro lingüístico es que el objeto preferente de estudio para el filósofo es el lenguaje, y que la metodología filosófica consiste en el análisis del lenguaje (de ahí la expresión «filosofía analítica»). Esto fue tomado muy en serio por los positivistas convirtiendo el análisis de conceptos como «espacio-tiempo», «evidencia», «confirmación», «explicación», «probabilidad», «objeto», etc., o la explicitación de las relaciones lógico-conceptuales entre los enunciados de la ciencia, como tareas defnitorias de la filosofía. ${ }^{18}$

Sin embargo, dada la diversidad de perspectivas coexistentes en el Positivismo Lógico, es difícil concretar una tesis compartida que vaya más allá de una mera proclama. La actitud antimetafísica confiere, sin duda, cierto

18 Siendo esta una tarea valiosa, sin duda, hay que decir que en las últimas tres décadas la filosofía actual ha purgado los excesos del giro lingüístico y se ha liberado de sus consecuencias paralizantes. Por tales consecuencias me refiero a la aversión no solo a la metafísica «especulativa», sino a la ontología en general, con el consiguiente riesgo de convertir a la filosofía en una reflexión metalingüística exclusivamente. Solamente entre las figuras secundarias del positivismo lógico encontramos alguna excepción a esta tendencia, por ejemplo, Gustav Bergmann. 
«aire de familia» a sus miembros, por recurrir a la expresión de Wittgenstein, pero ni es una marca distintiva del movimiento, pues había sido defendida anteriormente, ni fue entendida del mismo modo por unos u otros. En cuanto a la unidad de la ciencia, otra bandera común, también hubo diferencias sustanciales a la hora de entender y concretar dicha hipótesis de trabajo, según el sentido de unificación que cada autor consideró más relevante (conceptual, nomológica, metodológica,...). Otra aportación importante de este movimiento es el recurso sistemático a lenguajes formales («lenguajes lógicos») para resolver (o disolver) problemas filosóficos, aunque no todos los positivistas depositaron la misma confianza en el análisis formal; en toda la obra de Schlick, por poner un ejemplo destacado, apenas se encuentra nada de esto.

Sobre la vigencia actual de los enfoques formalistas no merece la pena extenderse. Basta observar el auge creciente en las últimas dos décadas de lo que se ha dado en llamar «Epistemología Formal» [Formal Epistemology]. ${ }^{19}$ No obstante, una parte importante del trabajo filosófico no recurre hoy por hoy a aparato formal alguno. También convendría añadir que, aun cuando la formalización pueda llegar a ser una ayuda decisiva, por sí sola no resuelve los problemas filosóficos, salvo contadas excepciones. Así pues, entender que el legado del positivismo lógico equivale a sacrificar toda orientación en la filosofía que no sea una orientación radicalmente formalista, no se sigue de lo que el movimiento fue en su conjunto.

Dicho esto, sí hay un presupuesto metafilosófico central para los positivistas que debe ser particularmente destacado. Me refiero al interés por la precisión y la claridad en la argumentación filosófica. La investigación del filósofo topa a menudo con la ambigüedad del discurso natural. Puede que ello obligue o aconseje recurrir a la formalización para lograr aquellos objetivos, con independencia de la parcela filosófica en la que nos ubiquemos. Lo importante, sin embargo, es que estos valores orientan el discurso y la investigación filosóficos sea cual sea el campo de trabajo. Ciertamente, no es que la claridad, la precisión y el rigor argumentativo sean objetivos que la reflexión filosófica persiga por sí mismos. No hay por qué suponer que realizarlos implica de suyo un rendimiento filosófico, una ganancia efectiva en la comprensión de los conceptos fundamentales del arte, la ciencia, la moral,... Son valores instrumentales, pues. Ahora bien, una de las enseñanzas del Positivismo Lógico, en mi opinión, es que esos valores son imprescindibles

19 Probablemente el centro más activo en este terreno sea hoy día el Munich Center for Mathematical Philosophy, fundado en 2010, y cuyos intereses abarcan ámbitos muy diversos dentro la filosofía. No deja de resultar irónico este camino de retorno a centroeuropa casi un siglo después del manifiesto fundacional del positivismo lógico. 
en la actividad filosófica. Posibilitan el análisis, la evaluación, la crítica y la refutación -en el sentido filosófico que tienen estos términos, esto es, en relación a argumentos. Imponen unas condiciones cuasi formales que, si no garantizan resultados filosóficamente iluminadores, si aseguran un mínimo exigible para que puedan obtenerse tales resultados. Con otras palabras, satisfacer esos valores no implica necesariamente un avance en filosofía; pero si no los respetamos, difícilmente conseguiremos algún triunfo.

Estas reflexiones finales permiten dotar de cierto contenido, aunque sea mínimo, a una noción capital que, si bien no se ha planteado abiertamente en este trabajo, subyace a toda la discusión metafilosófica de los positivistas lógicos, a saber, el «progreso filosófico». Desarrollar pormenorizadamente dicha noción excede en mucho los objetivos de este artículo. Me conformo con concluir que es en el modo de filosofar de los positivistas lógicos, y no tanto en alguna de sus tesis filosóficas particulares, donde puede atisbarse alguna respuesta al problema de fondo: el «estéril conflicto entre los sistemas filosóficos» en palabras de Schlick.

\section{REFERENCIAS BIBLIOGRÁFICAS}

AYER, A. J., 1936/1946. Language, Truth and Logic. Londres: Gollancz.

BUTTS, R. E., 1999. «The reception of German scientific Philosophy in NorthAmerica: 1930-1962)». En M. C. Galavotti y A. Pagnini, eds., Experience, Reality and Scientific Explanation. Dordrecht: Kluwer, pp. 1-14.

CARNAP, R., 1928. Der logische Aufbau der Welt. Berlin, Bernari (The Logical Structure of the World, Berkeley: University of California Press).

CARNAP, R., 1934a. Logische Syntax der Sprache, Wien: Springer Verlag. (The Logical Syntax of Language, Londres: Kegan Paul, 1937).

CARNAP, R., 1934b. «On the Character of Philosophic Problems», Philosophy of Science 1, pp. 5-19 [incluido posteriormente en The Linguistic Turn, antología editada en 1967 por Richard Rorty].

CARNAP, R., 1950/1962. The Logical Foundations of Probability. Chicago: The University of Chicago Press.

CARNAP, R., 1992. Autobiografia intelectual, Barcelona, Paidós. Es la traducción al castellano del prólogo de The Philosophy of Rudolf Carnap. P. Schilpp, ed., La Salle, Ill.: Open Court, 1962.

CARTWRIGHT, N., J. CAT, L. FLECK, T.E. UEBEL, 1996. Otto Neurath: Philosophy Between Science and Politics, Cambridge: Cambridge University Press.

CARUS, A. W. 2008. Carnap and Twentieth-Century Thought: Explication as Enlightenment. Cambridge, Cambridge University Press.

CAT, J., CHANG, H., N. CARTWRIGHT, 1995. «Otto Neurath: Politics and the Unity of Science». En P. Galison, D. Stump, eds., Disunity and Contextualism. Stanford: Stanford University Press, pp. 347-69.

CREATH, R., ed., 2012. Rudolf Carnap and the Legacy of Logical Empiricism. Dordrecht: Springer. 
Filosofía y Ciencia en el Positivismo Lógico ...

FRIEDMAN, M., 1999. Reconsidering Logical Positivism. New York: Cambridge University Press.

FRIEDMAN, M. y CREATH, R., eds., 2007. The Cambridge Companion to Carnap. Cambridge: Cambridge University Press.

FRIEDMAN, M., 2012. «Scientific Philosophy from Helmholtz to Carnap and Quine», en Creath, ed., pp. 1-11.

HARDCASTLE, G. L., A. RICHARDSON, eds., 2003. Logical Empiricism in North America (Minnesota Studies in the Philosophy of Science, vol. XVIII). Minneapolis: University of Minnesota Press.

HOLTON, G., 1993. «From the Vienna Circle to the Harvard Square: The Americanization of a European World Conception». En F. Stadler, ed., Scientific Philosophy: Origins and Developments. Dordrecht: Springer, pp. 47-74.

KOTERSKI, A., 2012. «The Backbone of the Straw Man. Popper's Critique of the Vienna Circle's Inductivism». En D. Dieks et al., eds., Probabilities, Laws and Structures. Dordrecht: Springer, pp. 457-468.

MILKOV, N. y V. PECKHAUS, eds., 2013. The Brlin Group and the Philosophy of Logical Empiricism. Dordrecht: Springer

MOULINES, U., 1996. «Las raíces epistemológicas del Aufbau de Carnap». En R. Cirera, A. Ibarra y Th. Mormann (eds.), El programa de Carnap. Ciencia, lenguaje, filosofía. Barcelona, Ediciones del Bronce, pp. 45-74.

NAGEL, E., 1936. «Impressions and Appraissals of Analytic Philosophy in Europe II». The Journal of Philosophy 33 (2), pp. 29-53.

NEURATH, O., 1931a. «Physikalismus». En Neurath 1983, pp. 52-57.

NEURATH, O., 1931b. «Soziologie im Physikalismus». En Neurath 1983, pp. 58-90.

NEURATH, O., 1932a. «Protoköllsatze». En Neurath 1983, pp. 91-99.

NEURATH, O., 1932b. «Unified Science and Psychology». En B. McGuinness, ed., Unified Science. Reidel, Dordrecht, 1987, pp. 1-23.

NEURATH, O., 1936a. «Individual Sciences, Unified Science, Pseudorationalism». En Neurath 1983, pp. 132-38.

NEURATH, O., 1936b. «Physicalism and the Investigation of Knowledge». En Neurath 1983, pp. 159-71.

NEURATH, O., 1937. «Unified Science and Its Encyclopedia». En Neurath 1983, 172-182.

NEURATH, O., 1941. «Universal Jargon and Terminology». En Neurath 1983, pp. 213-29.

NEURATH, O., 1944. Foundations of the Social Sciences, en O. Neurath, R. Carnap, Ch. Morris, eds., International Encyclopedia of Unified Science, vol. 2, n 1. Chicago: University of Chicago Press.

NEURATH, O., 1983. Philosophical Papers 1913-1946, R.S. Cohen y M. Neurath, eds. Dordrecht: Reidel.

PASSMORE, J., 1957. One Hundred Years of Philosophy. Londres, Duckworth.

PIHLSTRÖM, S., STADLER F. Y N. WEIDTMANN, 2017. Logical Empiricism and Pragmatism. Dordrecht: Springer.

REICHENBACH, H., 1936. «Logistic Empiricism in Germany and the Present State of its Problems». The Journal of Philosophy 33 (6), pp. 141-160. 
RICHARDSON, A., 1998. Carnap's construction of the world - The Aufbau and the emergence of logical empiricism. Cambridge: Cambridge University Press.

RORTY, R., 1967. The Linguistic Turn. Chicago, University of Chicago Press.

SCHLICK, M., 1930. «Die Wende der Philosophie». En Schlick 1979b, pp. 154-160.

SCHLICK, M., 1934. «Über das Fundament der Erkenntnis». En Schlick 1979b, pp. 370-387.

SCHLICK, M., 1936. «Meaning and Verification». En Schlick 1979b, pp. 339-369.

SCHLICK, M., 1979a. Philosophical Papers (vol. I), H. L. Mulder y B. F. van de Velde-Schlick, eds., Dordrecht: D. Reidel.

SCHLICK, M., 1979b. Philosophical Papers (vol. II), H. L. Mulder y B. F. van de Velde-Schlick, eds., Dordrecht: D. Reidel.

STADLER, F., 2001a. The Vienna Circle Studies in the Origins, Development, and Influence of Logical Empiricism. Dordrecht: Springer.

STADLER, F., 2001b. «The Philosophical and Political Pluralism of the Vienna Circle - The Example of Otto Neurath and Moritz Schlick». En Stadler, 2001a, pp. 277-284,

SUPPE, F., 1998. «Understanding Scientific Theories: An Assessment of Developments (1969-1998)». Philosophy of Science 67, pp. S102-S117.

UEBEL, Th., 2008. «Writing a Revolution: On the Production and Early Reception of the Vienna Circle's Manifesto». Perspectives on Science 16, pp. 70-102.

UEBEL, Th., 2012. «Carnap, Philosophy and Politics in its Broadest Sense», en R. Creath, ed., pp. 133-148.

UEBEL, Th., 2015. «Three challenges to the complementarity of the logic and the pragmatics of science». Studies in History and Philosophy of Science 53, pp. 23-32.

UEBEL, Th., 2018. «Carnap's Transformation of Epistemology and the Development of His Metaphilosophy». The Monist 101 (4), pp. 367-387.

WAISMANN, F., 1973. Wittgenstein y el Círculo de Viena. México, Fondo de Cultura Económica.

WOLÉNSKI, J., E. KÖHLER, eds., 1999. Alfred Tarski and the Vienna Circle: Austro-Polish Connections in Logical Empiricism. Dordrecht: Springer.

VALERIANO IrANZO es catedrático de Lógica y Filosofía de la Ciencia en la Universitat de València.

Líneas de Investigación:

Teoría bayesiana de la probabilidad, Filosofía de la estadística, Evidencia y explicación mecanicista, Realismo científico, Teorías de la justificación epistémica 
Publicaciones recientes:

(2020). «La crítica russelliana de la inferencia inductiva», en Gimeno-Simó, J. Luque, V. J., and Pérez-González, S. (eds.) Bertrand Russell: herencia y actualidad. Valencia, Tirant Lo Blanc (en prensa).

(2019) «Introduction: Explanation in science», Teorema vol. 38, n 3, 5-23.

Correo electrónico: iranzov@uv.es 
